\title{
Synthesis of novel benzothiazole compounds with an extended conjugated system
}

\author{
Peddiahgari Vasu Govardhana Reddy, Yang-Wei Lin, and Huan-Tsung Chang* \\ Department of Chemistry, National Taiwan University, Taipei-106, Taiwan \\ E-mail: changht@,ntu.edu.tw
}

\begin{abstract}
Novel cyano-substituted benzothiazoles (Schemes 1 and 2) were synthesized, which possess an extended conjugated system. Compound 6 consists of two benzothiazole units with bis(4vinylphenyl)acrylonitrile as a bridging group and compound $\mathbf{1 2}$ was two benzothiazole units and two dodecyloxy bis(4-vinylphenyl)-acrylonitrile units. Absorption and fluorescence properties were studied for compounds $\mathbf{6}$ and $\mathbf{1 2 .}$
\end{abstract}

Keywords: Benzothiazole, conjugated system, bis(4-vinylphenyl)acrylonitrile, disulfide, fluorescence

\section{Introduction}

Organic compounds with extended conjugated systems display unusual semiconducting and luminescent properties. ${ }^{1,2}$ In recent years, compounds with large two-photon absorption (TPA) cross-sections or two-photon-excited fluorescence (TPEF) cross-sections have received much research interest and have shown promising applications. ${ }^{3-9}$ The benzothiazolyl unit is an excellent acceptor that gives rise to donor- $\pi$-acceptor (D- $\pi$-A) type compounds. ${ }^{10-12}$ Benzothiazolyl derivatives have much better chemical, thermal, and photochemical stabilities than structurally similar compounds. ${ }^{10}$ 2-(3,5,6-Trifluoro-2-hydroxy-4-methoxyphenyl) benzothiazole with a zinc cation is used for fluorescent probe sensing. ${ }^{13}$ Bis-[2-(2hydroxyphenyl)benzothiazolate]zinc [Zn(BTZ)2] is one of the best white electroluminescent materials used in organic light-emitting diodes (OLEDs). ${ }^{14}$ Mintova et al. have synthesized nanosized zeolites by using a 2-(2'-hydroxyphenyl) benzothiazole precursor. ${ }^{15}$ Cyano-substituted poly-(phenylenevinylene) (PPV) has been used as an electron-transporting material in a highly efficient two layer light-emitting diode (LED) with PPV as a hole-transporting layer. ${ }^{16}$ These conjugated compounds which are accompanied by strong bathochromic shifts of the absorption and emission spectra showed increased electron affinity. ${ }^{17,18}$ Because of their rich electronic and 
photonic properties, conjugated organic molecules are ideal candidates as probe components for the structure of self-assembled monolayers (SAMs) that may find potential applications in optoelectronic devices. ${ }^{19}$ Yonezawa et al. synthesized gold nanoparticles by using a four-chained disulfide ligand and they concluded that the size of the metal core of gold nanoparticles could be finely tuned by the use of disulfide stabilizer molecules. ${ }^{20}$ The unsymmetrical azobenzene disulfide gold nanoparticles were synthesized in order to investigate the efficiency of azobenzene photoisomerization on colloidal gold surfaces. ${ }^{21}$

In addition, research on organic nanoparticles ${ }^{22-24}$ receives much attention in the fields of drug delivery, ${ }^{25}$ determinations of nucleic acids, ${ }^{26}$ and fluorescent ion sensors. ${ }^{27}$ Organic nanoparticles are brighter, photochemically stable, and water soluble. They also have high fluorescence quantum yields and a longer fluorescence lifetime than small organic molecules. ${ }^{27}$ In continuation of our research interest on fluorescence probe sensing, self-assembled monolayers and light emitting materials, we herein describe the synthesis and characterization of conjugated benzothiazole based chromophores (Schemes 1 and 2).

\section{Results and Discussion}

\section{Preparation}

The synthesis is described in Schemes 1 and 2. Starting, 2-[2-(4-methylphenyl)ethenyl]-1,3benzothiazole 3 was synthesized according to the procedure reported in the literature. ${ }^{28}$ The compound 3 was reacted with $N$-bromosuccinimide in dry benzene and subsequently it was reacted with aqueous sodium cyanide in tetrahydrofuran (THF) to form a benzothiazole vinyl phenyl acetonitrile 4. Similarly, compound 3 was reacted with $N$-bromo-succinimide and excess of aqueous hexamethylenetetramine in chloroform and then refluxed with a mixture of glacial acetic acid and water to obtain the benzothiazole vinyl benzaldehyde 5. The elaboration of the conjugated system of $\mathbf{6}$ was performed by reacing equimolar quantities of $\mathbf{4}$ and $\mathbf{5}$ in dry THF and tert-butyl alcohol at $50{ }^{\circ} \mathrm{C}$ while a small amount of tetrabutylammonium hydroxide was slowly dropped into the mixture. Similarly, bromododecyloxy substituted benzothiazolo acrylonitrile $\mathbf{1 1}$ was achieved by the condensation of bromododocyloxy benzaldehyde 9 with benzothiazolo acetonitrile $\mathbf{4}$ in dry THF and tert-butyl alcohol. The bromododecyloxy substituted benzothiazolo acrylonitrile $\mathbf{1 1}$ was reacted with hexamethyldisilathiane and tetra- $n$-butyl ammonium fluoride in dry THF to give the disulfide $\mathbf{1 2}$. The disulfide $\mathbf{1 2}$ was also synthesized from the benzothiazolo acetonitrile $\mathbf{4}$ and 4-bis(12-thiododecyloxy)dibenzaldyde $\mathbf{1 0}$ through another route, but in this method we obtained only a $5 \%$ yield. The structure of the synthesized derivatives was established by ${ }^{1} \mathrm{H}$ and ${ }^{13} \mathrm{C}$ NMR and MS measurements. 


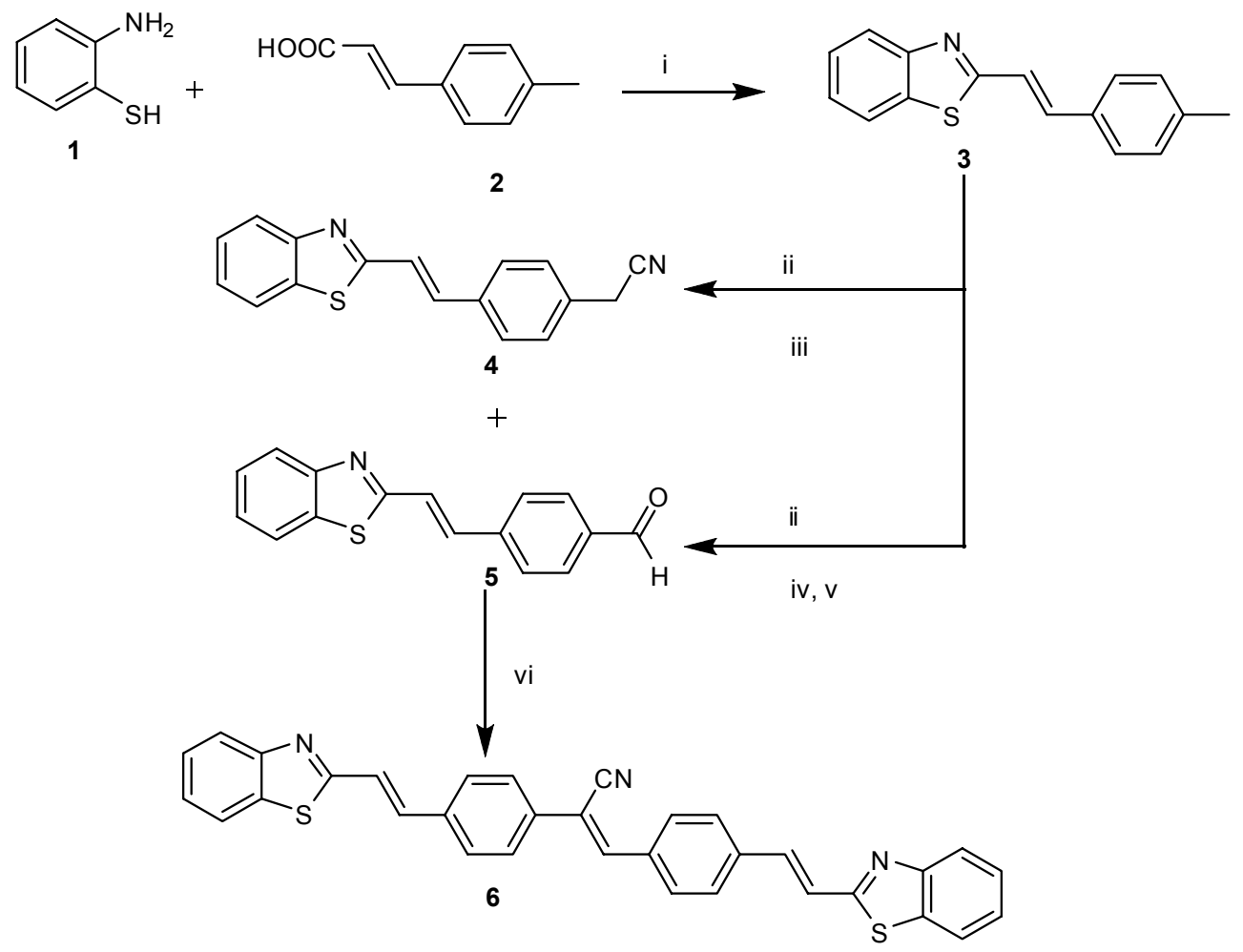

Scheme 1. Synthesis of cyano substituted conjugated benzothiazole 6. Reagents, conditions, and yields: i. $\mathrm{POCl}_{3}$, at reflux, $4 \mathrm{~h}, 80 \%$; ii. NBS, benzene, benzoyl peroxide; $10 \mathrm{~h}$; iii. $\mathrm{NaCN} / \mathrm{H}_{2} \mathrm{O}$, THF, $50^{\circ} \mathrm{C}, 24 \mathrm{~h}, 40 \%$; iv. $\mathrm{HMTA} / \mathrm{H}_{2} \mathrm{O}, \mathrm{CHCl}_{3}$, at reflux, 12h,; v. $\mathrm{AcOH} / \mathrm{H}_{2} \mathrm{O}$, at reflux, $2 \mathrm{~h}$, $25 \%$; vi. $t$ - $\mathrm{BuOH} / \mathrm{THF}, n-\mathrm{Bu}_{4} \mathrm{OH}, 1$ h, $96 \%$.

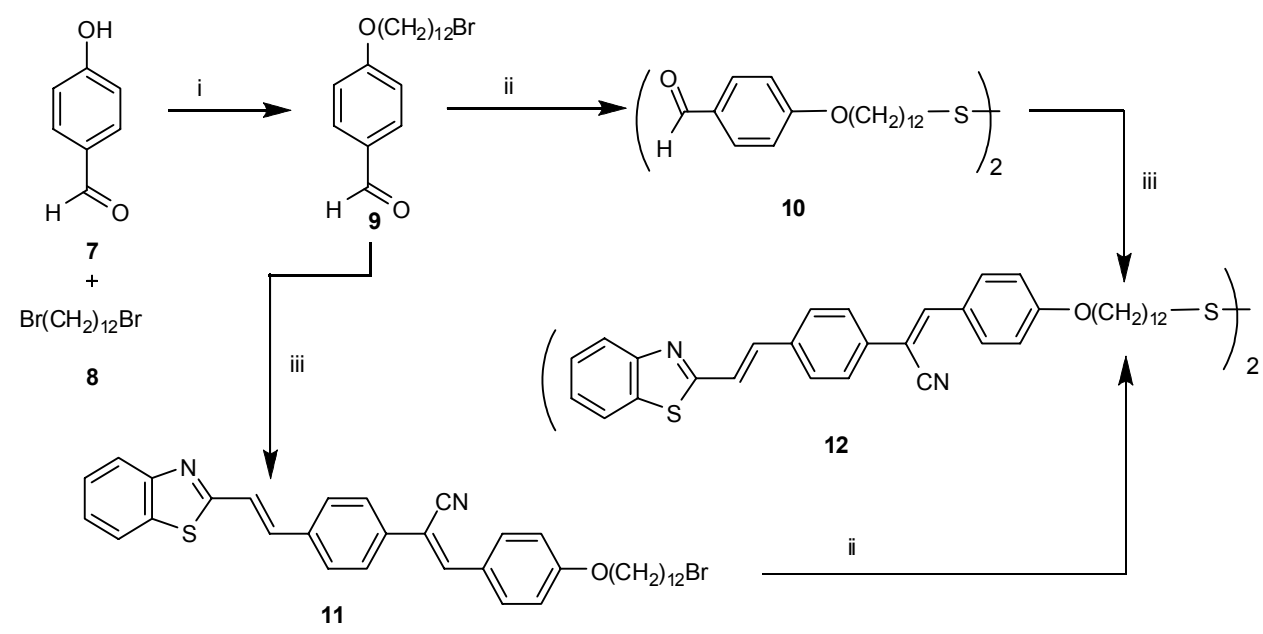

Scheme 2. Synthesis of cyano substituted conjugated benzothiazole dodecyloxy disulfide 12. Reagents, conditions, and yields: i. $\mathrm{KOH}, t-\mathrm{BuOH}$, at reflux, $20 \mathrm{~h}, 60 \%$; ii. $\left(\mathrm{Me}_{3} \mathrm{Si}\right)_{2} \mathrm{~S}$, TBAF, THF, $-10{ }^{\circ} \mathrm{C}$ to rt, $1 \mathrm{~h}, 95 \%$; iii. $4, t$-BuOH/THF, $n$ - $\mathrm{Bu}_{4} \mathrm{OH}, 50{ }^{\circ} \mathrm{C}, 1.5 \mathrm{~h}, 94 \%$. 


\section{Optical properties}

The fluorescence and UV-vis spectra were measured under ambient conditions. The THF solvent was HPLC grade for absorption and fluorescence mesurements. Concentrations of compounds were about $1 \times 10^{-5} \mathrm{M}$. The maximum absorption peaks of the bis $\{4-[(E)-2-($ benzo[ $d]$ thiazol-2yl)vinyl]phenyl\} acrylonitrile 6 and disulfide 12 are at $405 \mathrm{~nm}$ and $385 \mathrm{~nm}$, respectively (Figure 1). The absorption peak of $\mathbf{6}$ was shifted to the red, due to lengthing of a $\pi$-conjugated system. However, the absorbance of disulfide $\mathbf{1 2}$ was stronger than that of acrylonitrile $\mathbf{6}$, because of the more $\pi$-conjugated moiety in 12. Molar extinction coefficients $(\varepsilon)$ of acrylonitrile $\mathbf{6}$ and disulfide 12 at peak maximum position are $1.7 \times 10^{4}$ and $9.6 \times 10^{4}$, respectively.

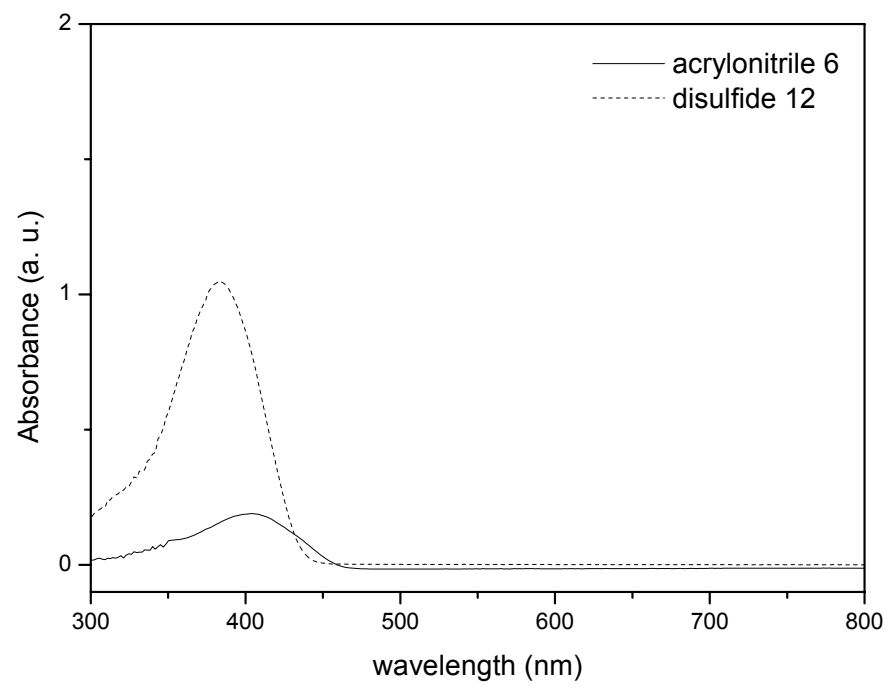

Figure 1. The UV-vis spectrum of acrylonitrile 6 and disulfide 12 in THF at $1 \times 10^{-5} \mathrm{M}$.

$\operatorname{Bis}\{4-[(E)-2-($ benzo $[d]$ thiazol-2-yl)vinyl $]$ phenyl $\}$ acrylonitrile $\mathbf{6}$ shows a fluorescence emission band at $495 \mathrm{~nm}$ while disulfide 12 shows an emission band at $434 \mathrm{~nm}$ in THF (Figure 2) at $10^{-5} \mathrm{M}$ concentration. The emission profile of $\mathbf{6}$ is very different from that of the disulfide 12, which may result from the presence of an extended $\pi$-conjugated length in $\mathbf{6}$. This can be explained by the electron-donor strength of the extra $\pi$-conjugated system. ${ }^{29,30}$ Additionally, the quantum yields $\left(\Phi_{\mathrm{f}}\right)$ of the two molecules vary considerably (see Table 1$)$. In these two molecules, acrylonitrile $\mathbf{6}$ showed the highest quantum yield. This can be attributed to a shorter conjugation path in the dodecyl analogue $\mathbf{1 2}$ compared to the extended conjugated acrylonitrile 6. 


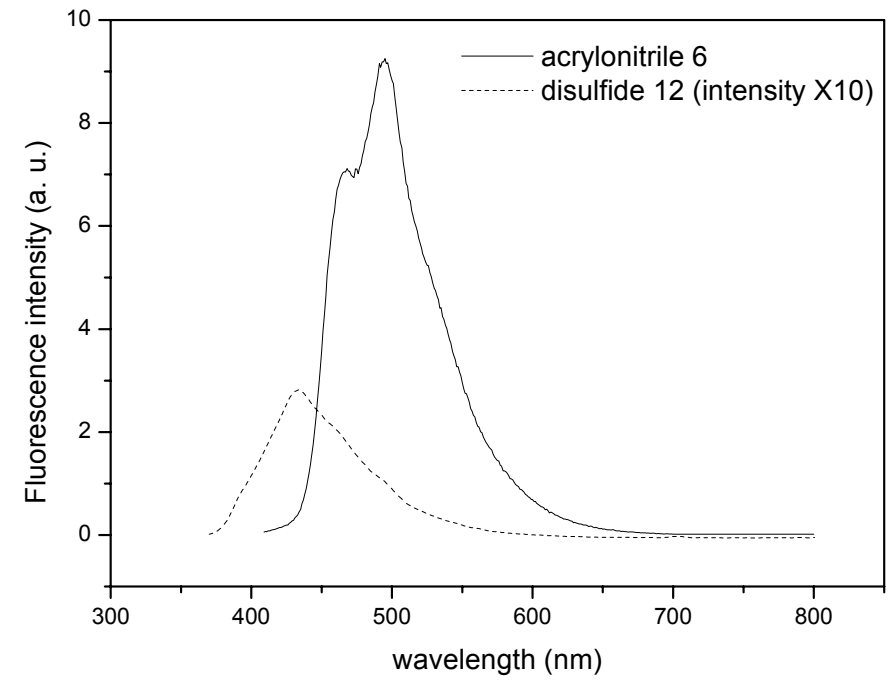

Figure 2. The fluorescence spectrum of acrylonitrile 6 and disulfide 12 in THF at $1 \times 10^{-5} \mathrm{M}$.

Table 1. Optical characteristic of the two compounds in $\mathbf{6}$ and $12 \mathrm{THF}^{\mathrm{a}}$

\begin{tabular}{lcccc}
\hline Molecule & $\lambda^{\mathrm{abs}}{ }_{\max } / \mathrm{nm}$ & $\varepsilon^{\mathrm{b}}$ & $\lambda^{\mathrm{f}}{ }_{\max } / \mathrm{nm}$ & $\Phi_{\mathrm{f}}(\%)^{\mathrm{c}}$ \\
\hline Compound 6 & 405 & $1.7 \times 10^{4}$ & 495 & 15 \\
Compound 12 & 385 & $9.6 \times 10^{4}$ & 435 & 0.08 \\
\hline
\end{tabular}

${ }^{\mathrm{a}}{ }^{\mathrm{abs}}{ }_{\max }, \lambda^{\mathrm{f}}{ }_{\text {max }}$ : Peak wavelength in the Uv-vis absorption and fluorescence spectra. ${ }^{\mathrm{b}} \varepsilon$ : Molar extinction coefficients at peak maximum position of two molecules. ${ }^{\mathrm{c}} 9,10$-diphenylanthracene was used as the reference $\left(\Phi_{\mathrm{f}}=90 \%\right.$ in cyclohexane $\left.{ }^{31}\right)$.

\section{Conclusions}

We have synthesized novel conjugated benzothiazoles that were characterized by ${ }^{1} \mathrm{H},{ }^{13} \mathrm{C} \mathrm{NMR}$, and MS data. Absorption and fluorescence properties were studied for the final compounds $\mathbf{6}$ and 12. Current work within the group is exploring the synthesis of benzothiazole-based organic nanoparticles. When compared to small organic molecules, the organic nanoparticles may possess greater stability, high quantum yields, and longer fluorescence lifetime. Thus, we expect that the organic nanoparticles that will be prepared will be suitable for sensing biomolecules such as proteins, DNA, and aminothiols. 


\section{Experimental Section}

General Procedures. The melting points were determined on a Fargo MP-2D Mel-Temp apparatus. Nuclear magnetic resonance spectra were recorded on a Bruker AM-400 $\mathrm{MHz}$ spectrometer. Mass spectra were obtained on a JEOL SX-102A spectrometer. A double-beam UV-vis spectrophotometer (Cintra 10e) obtained from GBC (Victoria, Australia) was used to measure the absorbance of compounds $\mathbf{6}$ and $\mathbf{1 2}$ and a fluorometer (Aminco Bowman) obtained from Thermo Spectronic (Pittsford, NY, USA) was used to collect the fluorescence spectra of compounds 6 and 12. 2-Aminothiophenol, 4-methylcinnamic acid, 1,12-dibromododecane and $\mathrm{N}$-bromosuccinimide were purchased from Acros Ltd. Hexamethyldisilathiane was obtained from the Aldrich Chemical Company.

Synthesis of 2-[2-(4-methylphenyl)ethenyl]-1,3-benzothizole (3). Benzothiazole 3 was synthesized according to the procedure reported in the literature. ${ }^{28} \mathrm{mp} 140-141{ }^{\circ} \mathrm{C}(\mathrm{EtOH}) ;{ }^{1} \mathrm{H}$ NMR (400 MHz, CDCl $)$ ): $7.98(\mathrm{~d}, J 8.1 \mathrm{~Hz}, 1 \mathrm{H}), 7.84(\mathrm{~d}, J 7.9 \mathrm{~Hz}, 1 \mathrm{H}), 7.50-7.19(\mathrm{~m}, 8 \mathrm{H}$, Ar$\mathrm{H}), 2.37\left(\mathrm{~s}, 3 \mathrm{H}, \mathrm{Ar}-\mathrm{CH}_{3}\right) ;{ }^{13} \mathrm{C} \mathrm{NMR}\left(100 \mathrm{MHz}, \mathrm{CDCl}_{3}\right): \delta 167.2,153.9,139.7,137.7,134.3$, 132.6, 129.6, 127.3, 126.2, 125.2, 122.8, 121.4, 121.1, 21.4; FAB MS: ( $m / z$, relative intensity) $252\left(\mathrm{M}^{+}+1,100 \%\right), 251\left(\mathrm{M}^{+}, 31\right), 154$ (66), $136(40), 107$ (18).

2-\{4-[(E)-2-(Benzo[d]thiazol-2-yl) vinyl] phenyl\}acetonitrile (4). A solution of 2-[2-(4methylphenyl)ethenyl]-1,3-benzothizole 3 (3.15 g, $12.5 \mathrm{mmol})$ and a small amount of benzoyl peroxide in freshly distilled benzene $(100 \mathrm{~mL})$ were added to the $N$-bromosuccinimide $(2.25 \mathrm{~g}$, $12.5 \mathrm{mmol}$ ). Then the mixture was refluxed for $10 \mathrm{~h}$. After cooling to room temperature, the precipitate was filtered off and the solvent was removed under vacuum. The residue was added into a solution of $\mathrm{NaCN}(2.45 \mathrm{~g}, 50 \mathrm{mmol})$ in aqueous tetrahydrofuran (THF) (40 mL). The mixture was then stirred at $50{ }^{\circ} \mathrm{C}$ for $24 \mathrm{~h}$. After being cooled to room temperature, the resulting mixture was separated using silica gel eluting with ethyl acetate/hexane (1:4) solvent. After the solvent was evaporated under vacuum, the title compound 4 (1.55 g, 40\%) was obtained as a yellow solid, ${ }^{28,32} \mathrm{mp} 169-170{ }^{\circ} \mathrm{C}$ (ethyl acetate/hexane); ${ }^{1} \mathrm{H}$ NMR (400 MHz, $\mathrm{CDCl}_{3}$ ): 7.99 (d, J $8.1 \mathrm{~Hz}, 1 \mathrm{H}), 7.97(\mathrm{~d}, J 7.8 \mathrm{~Hz}, 1 \mathrm{H}), 7.58-7.35(\mathrm{~m}, 8 \mathrm{H}), \delta 3.76(\mathrm{~s}, 2 \mathrm{H}) ;{ }^{13} \mathrm{C} \mathrm{NMR}(100 \mathrm{MHz}$, $\left.\mathrm{CDCl}_{3}\right): \delta 166.5,153.8,136.4,135.3,134.3,128.0,126.4,125.5,123.0,122.9,121.5,117.4$, 23.5; FAB MS: ( $m / z$, relative intensity) $277\left(\mathrm{M}^{+}+1,19 \%\right), 276\left(\mathrm{M}^{+}, 5\right), 267(7), 154(100), 136$ (70), 107 (21), 89 (19), 57 (35); MS: $\left(\mathrm{M}^{+}\right.$) (EI): Exact Mass Calcd for $\mathrm{C}_{17} \mathrm{H}_{12} \mathrm{~N}_{2} \mathrm{~S}, 276.0721$; Found 276.0942.

4-[(E)-2-(Benzo[d]thiazol-2-yl)vinyl]benzaldehyde (5). The bromination reaction of 2-[2-(4methylphenyl)ethenyl]-1,3-benzothizole $3(3.15 \mathrm{~g}, 12.5 \mathrm{mmol})$ was carried out by the same procedure as described above. The residue of bromination was added into a solution of hexamethylenetetramine (HMTA) $(7.0 \mathrm{~g}, 50 \mathrm{mmol})$ in aqueous chloroform $(40 \mathrm{~mL})$ and the mixture was refluxed for $12 \mathrm{~h}$. After being cooled to room temperature, the solvent was evaporated under reduced pressure. Then $50 \mathrm{~mL}$ of a glacial acetic acid $(\mathrm{AcOH})$ and a water mixture $(1: 1)$ was poured into the reaction mixture and refluxed for $2 \mathrm{~h}$. The resulting mixture 
was purified by column chromatography on silica gel using ethyl acetate/hexane (1:5) as eluent. After the solvent was removed under vacuum, the title compound 5 (0.82 g, 25\%) was obtained as a pale yellow solid, ${ }^{32} \mathrm{mp} 159-160{ }^{\circ} \mathrm{C}$ (lit. $\left.\mathrm{mp} 159.2-159.7{ }^{\circ} \mathrm{C}\right) ;{ }^{1} \mathrm{H}$ NMR $\left(400 \mathrm{MHz}, \mathrm{CDCl}_{3}\right)$ : $\delta 10.01(\mathrm{~s}, 1 \mathrm{H}), 8.02(\mathrm{~d}, J 8.1 \mathrm{~Hz}, 1 \mathrm{H}), 7.91-7.86(\mathrm{~m}, 3 \mathrm{H}), 7.72(\mathrm{~d}, J 8.2 \mathrm{~Hz}, 2 \mathrm{H}), 7.54-7.39(\mathrm{~m}$, $4 \mathrm{H}),{ }^{13} \mathrm{C} \mathrm{NMR}\left(100 \mathrm{MHz}, \mathrm{CDCl}_{3}\right): \delta 191.5,166.2,141.0,136.6,136.4,134.2,130.3,127.9$, 126.8, 126.0, 124.5, 123.0, 121.7, 52.9; FAB MS: ( $\mathrm{m} / \mathrm{z}$, relative intensity) $266\left(\mathrm{M}^{+}+1,10 \%\right)$, 265 (M+., 7), 221 (10), 206 (19), 192 (89), 154 (37), 149 (62), 136 (41), 95 (55), 69 (100), 55 (96).

(2Z)-2,3-Bis $\{4-[(E)-2-(b e n z o[d]$ thiazol-2-yl) vinyl]phenyl $\}$ acrylonitrile (6). A mixture of 2$\{4-[(E)-2-($ benzo$[d]$ thiazol-2-yl) vinyl] phenyl\}acetonitrile $4(0.28 \mathrm{~g}, 1 \mathrm{mmol})$ and 4-[(E)-2(benzo[d]thiazol-2-yl)vinyl]benzaldehyde $5(0.27 \mathrm{~g}, 1 \mathrm{mmol})$ in tert-butyl alcohol $(10 \mathrm{~mL})$ and THF (5 mL) was stirred at $50{ }^{\circ} \mathrm{C}$ for $1.5 \mathrm{~h}$. Tetrabutylammonium hydroxide (TBAH) (1 M solution in methanol) $(0.2 \mathrm{~mL})$ was slowly dropped into the mixture and stirred for $1 \mathrm{~h}$. The yellowish green precipitate was collected by filtration and washed with methanol to give the title compound $6(0.50 \mathrm{~g}, 96 \%)(\mathrm{EtOH}),{ }^{32,34} \mathrm{mp} 231-232{ }^{\circ} \mathrm{C} ;{ }^{1} \mathrm{H} \mathrm{NMR}\left(400 \mathrm{MHz}, \mathrm{CDCl}_{3}\right): \delta 7.96$, $7.82(\mathrm{dd}, J 7.4,7.7 \mathrm{~Hz}, 2 \mathrm{H}), 7.69(\mathrm{~d}, 8.0 \mathrm{~Hz}, 1 \mathrm{H}), 7.62-7.18(\mathrm{~m}, 8 \mathrm{H}) ;{ }^{13} \mathrm{C} \mathrm{NMR}(100 \mathrm{MHz}$, $\left.\mathrm{CDCl}_{3}\right): \delta 153.8,136.1,134.4,130.4,130.0,129.5,128.0,127.9,127.5,126.5,125.6,123.1$, 121.6; FAB MS: ( $m / z$, relative intensity) $524\left(\mathrm{M}^{+}+1,29 \%\right), 523\left(\mathrm{M}^{+}, 10\right), 460(7), 427$ (6), 369 (16), 341 (75), 307 (100), 289 (71), 267 (21), 259 (17), 239 (16), 211 (12); MS: (M ${ }^{+}$) (EI): Exact Mass Calcd for $\mathrm{C}_{33} \mathrm{H}_{21} \mathrm{~N}_{3} \mathrm{~S}_{2}, 523.1177$; Found 523.1826.

4-(12-Bromododecyloxy)benzaldehyde (9). To a stirred solution of 4-hydroxybenzaldehyde 7 (2.44 g, $20 \mathrm{mmol})$ in tert-butylalcohol $(40 \mathrm{~mL})$ was added 1, 12-dibromododecane 8 (18.0 g, 60 $\mathrm{mmol}$ ) and aqueous $\mathrm{KOH}(1.12 \mathrm{~g}, 20 \mathrm{mmol})$. The resulting mixture was heated to reflux for $20 \mathrm{~h}$. Water was then added and the impure product was extracted into $\mathrm{CH}_{2} \mathrm{Cl}_{2}$. The combined organic layers were dried over anhydrous $\mathrm{Na}_{2} \mathrm{SO}_{4}$, and the solvent was removed under vacuum. Excess dibromide was separated from product by column chromatography on silica gel with hexane as eluent and the product was eluted with ethyl acetate/ hexane (1:20). Evaporation of the solvent gave the title compound 9 (4.42 g, $60 \%$ ) as a white solid, ${ }^{35} \mathrm{mp} 57-58{ }^{\circ} \mathrm{C}$; ${ }^{1} \mathrm{H}$ NMR (400 MHz, $\left.\mathrm{CDCl}_{3}\right): \delta 9.85(\mathrm{~s}, 1 \mathrm{H}), 7.81(\mathrm{~d}, J 8.8 \mathrm{~Hz}, 2 \mathrm{H}), 6.97(\mathrm{~d}, J 8.6 \mathrm{~Hz}, 2 \mathrm{H}), 4.01(\mathrm{t}, J 6.5 \mathrm{~Hz}, 2 \mathrm{H}), 3.38$ $(\mathrm{t}, J 6.9 \mathrm{~Hz}, 2 \mathrm{H}), 1.84-1.77(\mathrm{~m}, 4 \mathrm{H}), 1.44-1.26(\mathrm{~m}, 16 \mathrm{H}) ;{ }^{13} \mathrm{C} \mathrm{NMR}\left(100 \mathrm{MHz}, \mathrm{CDCl}_{3}\right): \delta 190.7$, 164.2, 131.9, 129.7, 114.7, 68.4, 34.1, 32.8, 30.7, 29.6, 29.5, 29.4, 29.3, 29.0, 28.7, 28.1, 25.9; FAB MS: ( $m / z$, relative intensity) $371\left(\mathrm{M}^{+}+2,90 \%\right), 370\left(\mathrm{M}^{+}+1,37\right), 369\left(\mathrm{M}^{+}, 100\right), 307(19)$, 289 (7), 154 (79), 123 (17), 121 (26), 107 (32), 69 (50), 57 (81).

4,4'-(12,12'-Disulfanediylbis(dodecane-12,1-diyl)bis(oxy))dibenzaldehyde (10). Hexamethyldisilathiane $\left[\left(\mathrm{Me}_{3} \mathrm{Si}\right)_{2} \mathrm{~S}\right](0.435 \mathrm{~g}, 2.5 \mathrm{mmol})$ was added to the stirred solution of 4-(12bromododecyloxy)benzaldehyde $9(0.740,2 \mathrm{mmol})$ in dry THF $(10 \mathrm{~mL})$ at $-10{ }^{\circ} \mathrm{C}$. After stirring for 5 min tetra- $n$-butylamonium fluoride $(0.05 \mathrm{~mL}, 1 \mathrm{M}$ solution in THF) was added to the reaction mixture. Then this mixture was slowly warmed to room temperature and stirring was continued for an additional $1 \mathrm{~h}$. Dichloromethane was added and the organic layer was washed with saturated $\mathrm{NH}_{4} \mathrm{Cl}$ solution, dried over $\mathrm{MgSO}_{4}$, filtered and the solvent evaporated. The crude 
product was separated by flash column chromatography on silica gel with ethyl acetate /hexane (1:4). Evaporation of the solvent to afford the title compound $\mathbf{1 0}(1.24 \mathrm{~g}, 95 \%)$ as a white solid, ${ }^{36} \mathrm{mp} 77-78{ }^{\circ} \mathrm{C}$ (ethyl acetate /hexane); ${ }^{1} \mathrm{H}$ NMR (400 MHz, $\left.\mathrm{CDCl}_{3}\right): \delta 9.85(\mathrm{~s}, 1 \mathrm{H}), 7.81$ $(\mathrm{d}, J 8.7 \mathrm{~Hz}, 2 \mathrm{H}), 6.97(\mathrm{~d}, J 8.6 \mathrm{~Hz}, 2 \mathrm{H}), 4.01(\mathrm{t}, J 6.4 \mathrm{~Hz}, 2 \mathrm{H}), 2.65(\mathrm{t}, J 7.2 \mathrm{~Hz}, 1 \mathrm{H}), 2.47(\mathrm{t}, J$ $7.2 \mathrm{~Hz}, 1 \mathrm{H}), 1.78(\mathrm{t}, J 7.1 \mathrm{~Hz}, 2 \mathrm{H}), 1.64-1.23(\mathrm{~m}, 18 \mathrm{H})$; FAB MS: ( $\mathrm{m} / z$, relative intensity) 644 $\left(\mathrm{M}^{+}+2,44 \%\right), 643\left(\mathrm{M}^{+}+1,100\right), 642\left(\mathrm{M}^{+}, 32\right), 489$ (12), 391 (12), 354 (4), 321 (16), 289 (8), 199 (16), 154 (76), 123 (96), 107 (44).

\section{(2Z)-3-[4-(12-Bromododecyloxy)phenyl]-2-\{4-[(E)-2-(benzo[d]thiazol-2-yl)vinyl]-phenyl $\}$}

acrylonitrile (11). To a stirred solution of benzothiazolo acetonitrile $4(0.27 \mathrm{~g}, 1 \mathrm{mmol})$ and bromododocyloxy benzaldehyde $9(0.36 \mathrm{~g}, 1 \mathrm{mmol})$ in tert-butyl alcohol $(10 \mathrm{~mL})$ and THF (5 $\mathrm{ml}$ ) was heated at $50{ }^{\circ} \mathrm{C}$ for $1.5 \mathrm{~h}$. Tetrabutylammonium hydroxide (TBAH) (1 M solution in methanol) $(0.2 \mathrm{~mL})$ was added slowly into the mixture and stirred for $1 \mathrm{~h}$. The yellow precipitate was collected by filtration and washed with methanol to obtain the title compound $\mathbf{1 1}^{32,34}(0.58$ g, 94 \%), mp 122-123 ${ }^{\circ} \mathrm{C}(\mathrm{EtOH}) ;{ }^{1} \mathrm{H} \mathrm{NMR}\left(400 \mathrm{MHz}, \mathrm{CDCl}_{3}\right): \delta 7.99$ (d, J $\left.7.9 \mathrm{~Hz}, 1 \mathrm{H}\right), 7.88$ (t, $J$ 2H, 2H), 7.68-7.36 (m, 10H), $6.95(\mathrm{~d}, J 8.0 \mathrm{~Hz}, 2 \mathrm{H}), 4.01(\mathrm{t}, J 6.2 \mathrm{~Hz}, 2 \mathrm{H}), 3.40(\mathrm{t}, J 6.6 \mathrm{~Hz}$, $2 \mathrm{H}), 1.84-1.76(\mathrm{~m}, 4 \mathrm{H}), 1.46-1.26(\mathrm{~m}, 16 \mathrm{H})$; FAB MS: $\left(\mathrm{m} / \mathrm{z}\right.$, relative intensity) $628\left(\mathrm{M}^{+} \cdot+2\right.$, 100\%), $627\left(\mathrm{M}^{+}+1,75\right), 626\left(\mathrm{M}^{+}, 100\right), 579$ (20), 547 (12), 379 (4), 307 (98), 289 (50), 267 (10), 242 (16), 123 (96); MS: $\left(\mathrm{M}^{+}\right.$) (EI): Exact Mass Calcd for $\mathrm{C}_{36} \mathrm{H}_{39} \mathrm{~N}_{2} \mathrm{OSBr}, 626.1966$; Found 626.2102 .

\section{Bis(2Z,2'Z)-3,3'-(4,4'-(12,12'-disulfanediylbis(dodecane-12,1-diyl)bis(oxy))bis(4,1-pheny-} lene)bis(2-(4-((E)-2-(benzo[d]thiazol-2-yl)vinyl)phenyl)acrylonitrile) (12). Disulfide 12 was synthesized by the same procedure as 4-Bis(12-thiododecyloxy) dibenzaldyde 10. In another route, one equivalent of dibenzaldehyde $10(0.64 \mathrm{~g}, 1 \mathrm{mmol})$ was added into the two equivalents of benzothiazolo acetonitrile $4(0.55 \mathrm{~g}, 2 \mathrm{mmol})$ in tert-butyl alcohol $(10 \mathrm{~mL})$ and THF $(5 \mathrm{~mL})$. Then the reaction mixture was stirred at $50{ }^{\circ} \mathrm{C}$ for $1.5 \mathrm{~h}$. Tetrabutylammonium hydroxide (TBAH) (1 M solution in methanol) $(0.2 \mathrm{~mL})$ was slowly added into the mixture and stirred for 1 $\mathrm{h}$. The yellow precipitate was collected by filtration and washed with methanol to afford the title compound 12 (1.02 g, $89 \%$ ), mp 153-154 ${ }^{\circ} \mathrm{C}(\mathrm{EtOH}) ;{ }^{1} \mathrm{H} \mathrm{NMR}\left(400 \mathrm{MHz}, \mathrm{CDCl}_{3}\right): \delta 8.02(\mathrm{~d}, J$ $8.0 \mathrm{~Hz}, 1 \mathrm{H}), 7.89-7.84(\mathrm{~m}, 2 \mathrm{H}), 7.69-7.36(\mathrm{~m}, 10 \mathrm{H}), 6.96(\mathrm{~d}, J 8.8 \mathrm{~Hz}, 2 \mathrm{H}), 4.01(\mathrm{t}, J 6.5 \mathrm{~Hz}$, $2 \mathrm{H}), 3.38(\mathrm{t}, J 6.8 \mathrm{~Hz}, 2 \mathrm{H}), 1.86-1.75(\mathrm{~m}, 6 \mathrm{H}), 1.42-1.25(\mathrm{~m}, 14 \mathrm{H}) ;{ }^{13} \mathrm{C} \mathrm{NMR}(100 \mathrm{MHz}$, $\left.\mathrm{CDCl}_{3}\right): \delta 161.3,142.2,135.9,135.4,133.9,131.7,131.4,129.5,128.1,126.7,126.1,126.0$, $125.7,122.7,122.0,121.6,118.3,114.9,114.5,68.2,39.2,32.1,30.9,29.7,29.5,29.3,29.2$, 28.9, 26.0; FAB MS: (m/z, relative intensity) $1159\left(\mathrm{M}^{+}+1,20 \%\right), 1158\left(\mathrm{M}^{+}, 20\right), 127(36), 901$ (40), 869 (100), 607 (16), 579 (20), 359 (32), 341 (96), 307 (92), 289 (60), 242 (44); MALDITOF MS:1158.755 (M+., 46), 1126.775 (100), 578.640 (45), 546(50). 


\section{Acknowledgements}

This project was supported by the National Science Council, Taiwan (grant NSC-94 - 2120 -M 002 - 002, NSC 95-2113-M-002 -026 -MY3 and NSC 96-2627-M-002 -013) and is gratefully acknowledged.

\section{References}

1. Hohnholz, D.; Schweikart, K.-H. L.; Subamanian, R.; Hanack, M. Adv. Mater. 1999, 8, 646.

2. Hwang, D.-H.; Jang, M.-S.; Do, L.-M.; Chu, H. Y.; Shim, H. K.; Zyung, T. Mol. Cryst. Liq. Cryst. 1999, 327, 193.

3. He, G. S.; Bhawalkar, J. D.; Zhao, C. F.; Prasad, P. N. Appl. Phys. Lett. 1995, 67, 2433.

4. Ehrlich, J. E.; Wu, X. L.; Lee, I.-Y. S. Hu, Z.-Y.; Röckel, H.; Marder, S.R.; Perry, J. W. Opt. Lett. 1997, 22, 1843.

5. Ren, Y.; Fang, Q.; Yu, W. T.; Lei, H.; Tian, Y. P.; Jiang, M. H.; Yang, Q.C.; Mak, T. C. W. J. Mater. Chem. 2000, 10, 2025.

6. Denk, W.; Strickler, J. H.; Webb, W. W. Science 1990, 248, 73.

7. Meier, H. Angew. Chem. Int. Ed. 2005, 44, 2482.

8. Raposo, M. M. M.; Sousa, A. M. R. C.; Fonseca, A. M. C.; Krisch, G. Tetrahedron 2006, 62, 3493.

9. Tomava, R.; Petrova, P.; Stoycheva-Topalova, R.; Buroff, A.; Pirov, J. J. Optoelectron. Adv. Mater. 2007, 9, 501.

10. Kannan, R.; He, G. S.; Yuan, L. X.; Xu, F.; Prasad, P. N. Dombroskie, A. G.; Reinhardt, B.A.; Baur, J. W.; Vaia, R. A.; Tan, L.-S. Chem. Mater. 2001, 13, 1896.

11. Hobarik, P.; Sigmundova, I.; Zahradnik, P. Synthesis 2005, 600.

12. Rudrawar, S.; Kondaskar, A.; Chakraborti, A. K. Synthesis 2005, 2521.

13. Tanaka, K.; Kumagai, T.; Aoki, H.; Deguchi, M.; Iwata, S. J. Org. Chem. 2001, 66, 7328.

14. Yu, G.; Yin, S.; Liu, Y.; Shuai, Z.; Zhu, D. J. Am. Chem. Soc. 2003, 125, 14816.

15. Mintova, S.; Waele, V. D.; Hollzl, M.; Schmidhammer, U.; Mihailova, B.; Riedle, E.; Bein T. J. Phys. Chem. A. 2004, 108, 10640.

16. Greenham, N. C.; Moratti, S. C.; Bradley, R.; Friend, R. H.; Holmes, A. B. Nature 1993, 365 , 628.

17. Birckner, E.; Grummt, U.-W.; Rost, H.; Hartmann, A.; Pfeiffer, S.; Tillmann, H.; Hörhold, H.-H. J. Fluoresc. 1998, 8, 73.

18. Stalmach, U.; Detert, H.; Meier, H.; Gebhardt,V.; Haarer, D.; Bacher, A.; Schmidt, H.-W. Opt. Mater. 1998, 9, 77.

19. Doron, A.; Portnoy, M.; Mazzi, L.-D; Katz, E.; Willner, I. J. Am. Chem. Soc. 1996, 118, 8937.

20. Yonezawa, T.; Yasui, K.; Kimizuka, N. Langmuir 2001, 17, 271. 
21. Manna, A.; Chen, P.-L.; Akiyama, H.; Wei, T.-X.; Tamada, K.; Knoll, W. Chem. Mater. 2003, 15, 20.

22. Gong, X. C.; Milic, T.; Xu, C.; Batteas, J. D.; Drain,C. M. J. Am. Chem. Soc. 2002, 124, 14290.

23. Li, S.; He, L.; Xiong, F.; Li, Y.; Yang, G. J. Phys. Chem. B. 2004, 108, 10887.

24. Fu, H.-B.; Yao, J.-Y. J. Am. Chem. Soc. 2001, 123, 1434.

25. Lawrence, M. J. Eur. J. Drug Metab. Pharmacokinet. 1994, 3, 257.

26. Wang, L.; Dong, L.; Bian, G.; Xia, T.; Chen, H.; Cao, Q.; Li, L. Anal. Lett. 2004, 37, 1811.

27. Wang, L.; Wang, L.; Xia, T.; Dong, L.; Bian, G..; Chen, H. Anal. Sci. 2004, 20, 1013.

28. Cao, D. X.; Fang, Q.; Wang D.; Liu, Z.-Q.; Xue, G.; Xu, G.-B, Yu. W.-T. Eur. J. Org. Chem. 2003, 3628.

29. Yan, Y.; Tao, X.; Xu, G.; Zhao, H.; Sun, Y.; Wang, C.; Yang, J.; Yu, X.; Zhao, X.; Jiang, M. Aust. J. Chem. 2005, 58, 29.

30. Nicolai, S.-H.; Christensen, J. B.; Niles, H.; Thomas, B. J. Org. Chem. 2003, 122, 1275.

31. Hamai, S.; Hirayama, F. J. Phys. Chem. 1983, 87, 83.

32. An B.-K.; Kwon S.-K.; Jung, S.-D.; Park, S.Y. J. Am. Chem. Soc. 2002, 124, 14410.

33. Huang, Z.-L.; Lei, H.; Li, N.; Qui, Z.-R.; Wang, H.-Z.; Guo, J.-D.; Luo, Y.; Zhong, Z.-P.; Liu, X.-P. Zhou, Z.-H. J.Mat. Chem. 2003, 13, 708.

34. Detert, H.; Schollmeyer, D.; Sugiono, E. Eur. J. Org .Chem. 2001, 2927.

35. Kim, O.-K.; Fort, A.; Je, J.; Melinger, J. S. J. Am. Chem. Soc. 2006, 128, 4532.

36. Stellacci, F.; Bauer, C. A.; Timo, M.-F.; Wenseleers, W.; Marder, S.R.; Perry, J. W. J. Am. Chem. Soc. 2003, 125, 328. 